\title{
Politique
}

Politique

\section{Le contenu de la publicité télévisée des partis politiques au Québec}

\section{Denis Monière}

Numéro 22, automne 1992

URI : https://id.erudit.org/iderudit/040727ar

DOI : https://doi.org/10.7202/040727ar

Aller au sommaire du numéro

Éditeur(s)

Société québécoise de science politique

ISSN

0711-608X (imprimé)

1918-6584 (numérique)

Découvrir la revue

Citer cet article

Monière, D. (1992). Le contenu de la publicité télévisée des partis politiques au Québec. Politique, (22), 5-23. https://doi.org/10.7202/040727ar
Résumé de l'article

La recherche sur la publicité électorale au Québec est un terrain vierge. Dans cet article, l'auteur applique l'analyse lexicographique au contenu des messages publicitaires afin de comparer la publicité télévisée en langue française des deux principaux partis politiques du Québec diffusée durant la campagne électorale de 1989. En utilisant des indicateurs lexicaux, il examine le phénomène du mimétisme politique, celui de la personnalisation du message partisan ainsi que la stratégie de communication des partis. 


\title{
LE CONTENU DE LA PUBLICITÉ TÉLÉVISÉE DES PARTIS POLITIQUES AU QUÉBEC
}

\author{
Denis Monière \\ Universite de Montreal
}

La recherche sur la publicité électorale au Québec est un terrain vierge. Dans cet article, l'auteur applique l'analyse lexicographique au contenu des messages publicitaires afin de comparer la publicité télévisée en langue française des deux principaux partis politiques du Québec diffusée durant la campagne électorale de 1989. En utilisant des indicateurs lexicaux, il examine le phénomène du mimétisme politique, celui de la personnalisation du message partisan ainsi que la stratégie de communication des partis.

Dans une culture dominée par l'audiovisuel, la télévision devient un instrument indispensable des campagnes électorales. Ce médium permet à la fois de s'adresser à de vastes auditoires sociologiquement diversifiés et d'atteindre les électeurs les moins informés et les moins politisés'. Patrick Devlin estime qu'aux Etats-Unis les publicités télévisées influencent entre 10 et $20 \%$ de l'électorat, et particulièrement les électeurs qui sont indécis².

Les partis misent sur la publicité télévisée parce que l'achat de temps d'antenne, même s'il est coûteux, est le moyen de contrôler le contenu des messages diffusés, le moment et la fréquence de leur transmission et, dans une certaine mesure, de viser des clientèles électorales. Les partis n'ont pas la même emprise sur les autres supports de communication, tels que les conférences de presse ou les reportages journalistiques, dans

1. Une enquête effectuée lors des élections fédérales de 1988 a établi que $59,7 \%$ des 3616 personnes interrogées avaient vu les messages publicitaires des partis. Voir R. Johnston, A. Blais, H. Brady, J. Crête, Canadian Election Study, Institute for Social Research, université York.

2. Voir «An Analysis of Political Television Commercials, 1952-1984" dans L. L. Kaid, D. Nimmo et al, New Perspectives on Political Advertising, Carbondale, Southern Illinois University Press, 1986, p. 22.

Revue québécoise de science politique, no 22, automne 1992. 
lesquels le message est soumis à l'interprétation des journalistes ou au découpage et au montage des prises de vue.

Les messages télévisés de courte durée, c'est-à-dire de 15 ou de 30 secondes, ont aussi l'avantage de réussir parfois à briser le mur de l'exposition sélective. Insérés parmi d'autres messages publicitaires dans une émission très populaire, ils suscitent peu de réactions de rejet. Ils rejoignent des clientèles qui, normalement, chercheraient à fuir le discours politique soit parce qu'elles ne s'intéressent pas à ce domaine, soit parce qu'elles ne partagent pas les idées du parti qui expose ses thèses.

Selon plusieurs enquêtes menées dans différents pays ${ }^{3}$, la fonction de la publicité télévisée n'est pas de convaincre l'électeur, de l'amener à changer son choix de vote. Elle sert plutôt à renforcer les convictions partisanes et à attirer l'attention des électeurs sur les principaux enjeux de l'élection. En définissant l'image de marque d'un parti et en fixant ce qu'on appelle les "priorités de l'agenda politique», elle oriente la réflexion de l'électeur et influence indirectement son choix. Elle peut aussi servir à faire connaître un candidat ou à corriger son image, si celle-ci est négative; elle permet également d'attaquer les partis adverses ${ }^{4}$. Enfin, la publicité électorale informe les électeurs sur les orientations des partis ${ }^{5}$.

Paterson et McClure ont observé que l'impact des messages publicitaires varie selon le degré $d^{\prime}$ information des citoyens : moins les citoyens sont informés, plus le message peut influencer leur perception des enjeux électoraux. Chez les gens bien informés ou politisés, la publicité contribue surtout à cristaliser plus fortement les opinions qu'ils ont déjà. Les messages publicitaires agissent également sur les militants et particulièrement sur les travailleurs

3. Voir Blumler et al., La télévision fait-elle l'élection ?, Paris, Fondation nationale des sciences politiques, 1976; E. Diamond et S. Bates, The Spot: the Rise of Political Advertising on Television, Chicago, MIT Press, 1988; T. Paterson et R. McClure, The Unseing Eye, New York, Putnam's, 1976; P. Devlin, «An Analysis of Presidential Television Commercials», dans L. L. Kaid, D. Nimmo, K. R. Sanders, New Perspectives on Political Advertising, Carbondale, Southern Illinois University Press, 1986, p. 21-54.

4. Voir M. Kern, 30-Second Politics, New York, Praeger, 1989, p. 6.

5. Voir Doris Graber, Mass Media and American Politics, Washington, Congressional Quarterly, 1989, p.196. 
d'élection en stimulant leur ardeur et en augmentant leur confiance dans la victoire de leur parti ${ }^{6}$.

La publicité électorale au Québec est encore un terrain vierge pour la recherche. Afin d'ouvrir des pistes, il nous a semblé pertinent de nous intéresser d'abord au contenu des messages publicitaires télévisés et de comparer la publicité en langue française, émise par les deux principaux partis politiques du Québec durant la campagne électorale de 1989?.

L'analyse lexicale a jusqu'ici été peu souvent appliquée au contenu des messages politiques. On s'en est servi surtout pour dépouiller des corpus considérables comme, par exemple, les résolutions des congrès syndicaux en France ${ }^{8}$ ou pour étudier le vocabulaire de penseurs et de leaders politiques ${ }^{9}$ ou encore le contenu de journaux révolutionnaires ${ }^{10}$. Quant aux travaux sur la publicité électorale, effectués aux État-Unis, ils s'intéressent plus aux réactions suscitées par les messages qu'à leur substance proprement dite. L'analyse de contenu, pour sa part, sert surtout à décrire des messages codifiés à partir d'une grille de critères préétablis.

L'analyse lexicale, qui procède plutôt de la logique inductive, se distingue de l'analyse de contenu par un traitement systématique du texte et par l'objectivité de la grille d'analyse et de la procédure de codification. Elle a l'avantage d'être plus fiable sur le plan empirique, mais elle a l'inconvénient de conduire à des conclusions dont la portée théorique est plus limitée.

6. Voir K. G. Sheinkopf, «How Political Party Workers Respond to Political Advertising", dans Journalism Quarterly, vol. 50, été 1973, p. 334-339.

7. Les élections furent déclenchées le $\mathbf{9}$ août. Le scrutin eut lieu le 25 septembre 1989.

8. Voir «Discours syndical ouvrier en France», dans Mots, $n^{\circ} 14$, mars 1987.

9. Voir A.-M. Chouillet, "Vocabulaire politique de Diderot et de quelques encyclopédistes", dans Mots, $n^{\circ} 1$, oct. 1980; J.-M. Cotteret, Le vocabulaire du général de Gaulle, Paris, Presses de la Fondation nationale des sciences politiques, 1969; Dominique L'Abbé, Le vocabulaire de François Mitterrand, Paris, Presses de la Fondation nationale des sciences politiques, 1990.

10. Voir André Salem, "Approches du temps lexical», dans Mots, oct. 1988, p. 105-143. 
En utilisant des indicateurs lexicaux, nous tenterons de répondre à certaines questions générales propres à l'analyse des discours électoraux :

1- Les partis, lorsqu'ils s'adressent à des auditoires peu politisés et différenciés sociologiquement, mettent-ils une sourdine à leur idéologie ? Cherchent-ils à se rapprocher de l'électeur médian ? Ont-ils tendance à pratiquer ce qu'on appelle le mimétisme politique ou, au contraire, affirment-ils leurs différences avec les autres partis?

2- La communication télévisuelle renforce-t-elle la tendance à la personnalisation du pouvoir en accordant une grande importance aux leaders, au détriment de l'identification partisane ?

3- Le contenu des messages publicitaires est-il fonction de la position des partis dans le système partisan ? Autrement dit, le parti au pouvoir adopte-t-il une stratégie de valorisation et le parti d'opposition opte-t-il pour une stratégie offensive?

\section{Méthode d'analyse}

Nous avons constitué le corpus des discours publicitaires en enregistrant sur bandes vidéo toutes les émissions télédiffusées du 28 août au 24 septembre $1989^{11}$ entre 18 heures et 24 heures, sur quatre chaînes montréalaises : deux francophones, soit RadioCanada et Télémétropole et deux anglophones, soit CBC et CTV. Nous avons extrait tous les messages publicitaires et identifié pour chacun les émissions dans lesquelles ils étaient diffusés afin de pouvoir par la suite déterminer les cotes d'écoute potentielles. Nous avons aussi retranscrit le contenu de chaque message en identifiant les divers locuteurs ${ }^{12}$. Ayant opté pour une perspective comparative, nous avons dû exclure de l'analyse les messages diffusés en anglais parce que seul le Parti libéral du Québec (PLQ) a acheté du temps d'antenne sur les chaînes anglophones. Enfin, les autres partis n'ayant pas fait usage de publicité payante, nous n'avons pu inclure leurs messages dans notre étude.

11. Ce sont les dates où la télédiffusion des messages publicitaires est autorisée par la loi électorale du Québec.

12. Ce corpus n'inclut pas nécessairement tous les messages diffusés par les partis durant la campagne électorale, car certains d'entre eux ont pu etre présentés en dehors des heures d'observation choisies. 
Le Parti québécois a diffusé un message de 4 minutes, 6 messages de 15 secondes et 2 messages de 30 secondes; le Parti libéral du Québec a, pour sa part, diffusé 5 messages de 4 minutes et 5 messages de 30 secondes. Comme, en publicité, la rétention du message dépend en partie de sa répétition, le contenu des publicités n'est pas entièrement différent ou original. Certaines phrases sont reprises intégralement d'un message à l'autre, ce qui est évidemment le cas des slogans et des thèmes musicaux. Ce taux de redondance (nombre de phrases répétées dans au moins deux messages, divisé par le nombre total de phrases) est de 27,3 \% pour le Parti québécois et de 31 \% pour le Parti libéral.

Tableau I : Caractéristiques du corpus

\begin{tabular}{||l|c|c||}
\hline & Parti libéral & Parti québécois \\
\hline \hline Nombre total de mots & 2930 & 1007 \\
Mots distincts & 732 & 300 \\
$\%$ & 25 & 29.8 \\
Nombre de phrases & 171 & 77 \\
Phrases répétées & 53 & 21 \\
Nombre de mots dans & 17 & 13 \\
les phrases & & \\
\hline
\end{tabular}

Nous avons utilisé deux unités d'analyse différentes pour comparer les messages : le mot, défini comme l'ensemble des caractères entre deux espaces blancs, et la phrase qui est l'ensemble des mots compris entre deux points (voir tableau I). Pour effectuer l'analyse lexicale, nous avons employé le logiciel SPAD.T qui permet d'obtenir la fréquence des mots et celle des segments répétés, et de déterminer les mots les plus caractéristiques de chaque discours publicitaire ${ }^{13}$. Par ailleurs, chaque phrase a été codifiée selon les types d'énoncés suivants : jugements de fait (description de situation ou de problème), objectifs, performance passée, qualité du chef, du parti ou des candidats, critiques de l'adversaire, appel aux électeurs, slogan. Par définition, un message publicitaire doit être simple, c'est-à-dire

13. Voir André Salem et L. Lebar, Analyse statistique des données textuelles, Paris, Dunod, 1988. Le logiciel SPAD.T version 1989 est produit par le CISIA et est utilisé sur micro IBM. 
composé de phrases courtes et ne contenant qu'un type d'énoncé. Nous avons classé les énoncés qui diffèrent de cette règle dans la catégorie de l'énoncé le plus long (selon le nombre de mots prononcés).

Comme le message publicitaire est entièrement réglé par le parti et que rien n'y est laissé au hasard, il se prête bien à l'analyse quantitative, puisque, par définition, le choix des mots et leur fréquence sont voulus et sont particulièrement significatifs.

\section{La diffusion des messages}

Avant d'aborder l'analyse du vocabulaire, nous comparerons les stratégies de diffusion des messages, qui peuvent révéler des différences significatives entre les partis.

Alors que la loi électorale québécoise réglemente de façon rigoureuse le financement des partis politiques afin de favoriser un meilleur équilibre dans la compétition électorale, elle est beaucoup moins contraignante quant aux règles d'accès aux médias électroniques. À la différence du fédéral, le Québec n'a pas la compétence pour obliger les radiodiffuseurs à accorder du temps d'antenne gratuit aux partis politiques. Dès lors, la loi stipule simplement, à l'article 423, que tout radiodiffuseur "peut mettre gratuitement à la disposition des chefs des partis et des candidats du temps d'émission à la radio ou à la télévision(...) pourvu qu'il offre un tel service de façon équitable». La seule règle qu'impose la loi aux partis et aux diffuseurs est d'interdire toute dépense de publicité avant le vingt-neuvième jour précédant celui du scrutin (article 429). Les dépenses de publicité sont donc laissées à la discrétion des partis, mais elles sont toutefois limitées par le plafond des dépenses électorales que ceux-ci doivent respecter.

Tableau II : Répartition des types de messages

\begin{tabular}{||l|c|c|c|c||}
\hline & PLQ & PQ & NPD & Verts \\
\hline \hline Pub. gratuite & 10 & 9 & 3 & 2 \\
4 minutes (R-C) & & & & \\
Pub. payante (TVA) & 0 & 42 & 0 & 0 \\
15 sec. & $77^{*}$ & 37 & 0 & 0 \\
$30 \mathrm{sec}$ & 87 & 88 & 3 & 2 \\
\hline TOTAL &
\end{tabular}

* De ce nombre 23 ont été diffusés en anglais à CTV. 
Le combat publicitaire ne se fait donc pas à armes égales et les disparités de ressources entre les partis peuvent créer un déséquilibre dans le pouvoir d'influence.

Le tableau II indique la répartition des types de messages entre les partis. Seule la Société Radio-Canada a offert aux partis du temps d'émission gratuit, réparti sensiblement de façon égale entre les deux principaux partis malgré l'écart important entre le pourcentage de votes obtenus par le Parti libéral (56\%) et par le Parti québécois $(38 \%)$ à la dernière élection. Par ailleurs, la société d'état a offert du temps gratuit au NPD et au Parti vert, même s'ils n'avaient pas obtenu $3 \%$ des votes en 1985 et n'avaient aucun député à l'Assemblée nationale. Les autres tiers partis n'ont pas eu droit à ce traitement de faveur.

Forts de ce temps gratuit sur le réseau public où ils étaient assurés d'une bonne exposition, les partis en ont profité pour investir la totalité de leur budget publicitaire dans les réseaux privés (TVA et CTV pour le Parti libéral qui a acheté le temps de diffusion de 23 messages de 30 secondes en anglais).

\section{La place des messages}

Les partis ont adopté des stratégies de diffusion différentes. Entre le 31 août et le 7 septembre, le Parti québécois a misé sur des messages de 15 secondes diffusés deux par deux dans la même plage publicitaire; par la suite il a émis des messages de 30 secondes. Le Parti libéral s'en est tenu à des messages de 30 secondes.

Tableau III : Répartition hebdomadaire des publicités

\begin{tabular}{||l|r|r|r|c||}
\hline \multirow{2}{*}{} & \multicolumn{2}{|c|}{ Parti libéral } & \multicolumn{2}{c|}{ Parti québécois } \\
\cline { 2 - 5 } & $\mathrm{N}$ & $\%$ & $\mathrm{~N}$ & $\%$ \\
\hline \hline 31 août - 5 sept. & 9 & 10,3 & 30 & 34,5 \\
6 sept. - 11 sept. & 14 & 16,1 & 16 & 18,4 \\
12 sept. - 17 sept. & 32 & 36,8 & 21 & 24,1 \\
18 sept. - 23 sept. & 32 & 36,8 & 20 & 23,0 \\
\hline Total & 87 & & 87 & \\
\hline
\end{tabular}


Comme le tableau III le montre, le Parti québécois a choisi d'utiliser ses ressources publicitaires au début de la campagne afin de mobiliser ses soutiens. Le Parti libéral a plutôt déployé son offensive durant les deux dernières semaines.

Nous avons aussi pu constater que, contrairement à une idée reçue, il n'y a pas eu de matraquage publicitaire dans les derniers jours de la campagne. Selon notre relevé, en effet, dans les trois derniers jours, la moyenne quotidienne de messages diffusés fut de 3 pour le Parti québécois et de 4,3 pour le Parti libéral alors que la moyenne quotidienne pour l'ensemble de la campagne fut de 3,5 pour les deux partis.

La fréquence de diffusion de chaque message est un autre facteur de rétention. Là encore, nous avons observé des différences : certains messages ont été diffusés plus souvent que les autres, les partis choisissant de privilégier certains thèmes ou enjeux. Ainsi, les deux messages les moins diffusés par le Parti québécois portaient sur la constitution (4 fois) et sur l'agriculture ( 5 fois). Les plus fréquents furent ceux où Jacques Parizeau parlait de son amour du Québec ( 21 fois) et celui où il présentait son équipe (16 fois). Ces deux messages de 30 secondes ont été présentés à la fin de la campagne, soit à partir du 11 septembre pour le premier et du 18 septembre pour le second ${ }^{14}$. Par leur contenu, ils cherchaient à rallier les électeurs indécis en jouant sur I'identité québécoise.

Le Parti libéral, pour sa part, a produit 5 messages différents de 30 secondes. Le premier, qui portait sur le bilan du gouvernement, a été diffusé 5 fois au début de la campagne, soit du 31 août au 4 septembre; les messages sur la qualité de vie ont été diffusés 12 fois, et les messages sur la croissance économique, émis 6 fois, ont pris le relais du 4 au 13 septembre. Puis le Parti libéral a diffusé 19 fois un message attaquant le projet d'indépendance du Parti québécois. Enfin, un message vantant les mérites de l'équipe libérale a paru 12 fois, du 19 au 23 septembre.

Afin d'évaluer les cotes d'écoute potentielles de ces messages, nous avons eu recours au sondage BBM effectué dans la semaine du 14 au 20 septembre. En mettant en relation les heures de diffusion des messages et les cotes obtenues par les émissions où ils ont été diffusés, nous avons pu observer que deux tiers des messages ont été présentés dans des émissions dont les

14. Les autres messages du Parti québécois abordaient les thèmes suivants: la famille 6 fois, la santé 7 fois, l'environnement 8 fois, la langue 10 fois. 
cotes d'écoute étaient supérieures à 500000 téléspectateurs. A cet égard, les deux partis ont suivi la même stratégie en concentrant la très grande majorité de leurs messages durant la semaine et en négligeant la fin de semaine où les cotes sont plus faibles. Curieusement, le lundi qui était la meilleure journée à TVA n'a pas obtenu la faveur des deux partis qui ont préféré les mercredis et jeudis.

Les deux partis se distinguent toutefois dans le choix des plages horaires : le Parti libéral a eu tendance à concentrer ses messages au début et à la fin de la soirée, en les rattachant principalement aux émissions d'information. Le Parti québécois a préféré le milieu et la fin de la soirée. Quoi qu'il en soit, les deux partis ont eu tendance à présenter leurs messages pendant le journal télévisé de 23 heures, soit $36 \%$ des messages du Parti libéral et $42 \%$ de ceux du Parti québécois. Ce choix peut s'expliquer de trois façons : le temps publicitaire des téléromans est réservé longtemps à l'avance pour les produits commerciaux; les émissions d'information ont un auditoire plus restreint que les téléromans, mais ces émissions sont aussi moins victimes de l'exposition sélective; enfin, le message publicitaire situé dans ce contexte vient renforcer l'impact de l'événement médiatique de la journée, présenté au cours du journal télévisé.

\section{La thèse du mimétisme}

Le discours publicitaire devrait justement permettre de vérifier la thèse du mimétisme des partis. En effet, les messages publicitaires visant surtout les indécis, les partis, en principe, ne devraient pas trop chercher à se distinguer les uns des autres et auraient intérêt à se rapprocher de l'électeur médian. Afin de vérifier l'exactitude de cette thèse, nous comparerons les contenus des messages publicitaires en les soumettant à l'analyse lexicale qui identifie les mots les plus fréquemment employés et les mots les plus caractéristiques de chaque discours publicitaire. La comparaison du vocabulaire révèle les thèmes les plus récurrents dans le discours et peut servir à ce titre d'indicateur objectif de ressemblance ou de différence.

Selon nos observations, le Parti libéral donne une plus grande importance aux arguments quantifiés : on retrouve 45 chiffres dans ses messages comparativement à 4 seulement dans ceux du Parti québécois. Cet écart peut s'expliquer par la nécessité pour le parti gouvernemental d'insister sur ses réalisations et $d$ 'adopter des procédés qui frappent l'imagination 
comme la citation de chiffres impressionnants. Les dates occupent aussi une place essentielle dans un discours bilan (21 sur 45 ) où il faut indiquer des repères chronologiques pour bien mettre en évidence la performance du gouvernement. Des segments de phrases comme "Qui aurait dit, il y a quatre ans, qu'on aurait..." répété 6 fois, ou encore "quand on a un tel bilan" répété 4 fois, illustrent cette tactique. L'écart entre les partis traduit aussi une différence d'image projetée par les deux partis, le Parti libéral ayant toujours donné la priorité à l'économie et le Parti québécois se présentant plutôt comme un parti d'idées.

La comparaison du vocabulaire dans le tableau IV confirme cette divergence dans les messages des partis.

Tableau IV : Tableau comparatif des lexiques publicitaires des partis $^{15}$

\begin{tabular}{||l|r|r||}
\hline Noms & $\mathrm{PL}$ & $\mathrm{PO}$ \\
\hline \hline ans & 26 & 2 \\
avenir & 29 & 2 \\
bilan & 11 & 0 \\
chômage & 7 & 0 \\
croissance & 7 & 0 \\
déficit & 5 & 0 \\
développement & 8 & 0 \\
dollars & 17 & 0 \\
économie & 22 & 3 \\
emplois & 14 & 10 \\
environnement & 10 & 1 \\
équipe & 13 & 1 \\
gouvernement & 29 & 2 \\
impôts & 9 & 0 \\
investissements & 20 & 0 \\
jeunes & 18 & 2 \\
libéraux & 0 & 11 \\
libéral & 30 & 0 \\
parti & 11 & 34 \\
Québec & 57 & 40 \\
québécois & 14 & 11 \\
québécoise & 10 & 0 \\
\hline \hline Pronoms & $\mathrm{PL}$ & $\mathrm{PQ}$ \\
\hline \hline je me & 13 & 32 \\
il(s) & 39 & 13 \\
nous & 46 & 15 \\
nos & 10 & 3 \\
notre & 27 & 3 \\
qui & 48 & 10 \\
on & 61 & 27 \\
\hline \hline
\end{tabular}

15. Nous avons retenu uniquement les termes qui revenaient 5 fois et plus. 


\begin{tabular}{|c|c|c|}
\hline Verbes ${ }^{\circ}$ & PL & PQ \\
\hline aller & 11 & 2 \\
\hline avoir & 80 & 33 \\
\hline assurer & 20 & 0 \\
\hline continuer & 5 & 1 \\
\hline dire & 11 & 2 \\
\hline devoir & 5 & 3 \\
\hline être & 80 & 30 \\
\hline faire & 32 & 19 \\
\hline falloir & 13 & 4 \\
\hline grandir & 7 & 0 \\
\hline laisser & 0 & 8 \\
\hline marcher & 10 & 0 \\
\hline parler & 1 & 7 \\
\hline pouvoir & 12 & 7 \\
\hline prendre & 0 & 17 \\
\hline réduire & 7 & 0 \\
\hline voter & 15 & 8 \\
\hline vouloir & 6 & 0 \\
\hline Adverbes & PL & $P Q$ \\
\hline avec & 22 & 4 \\
\hline beaucoup & 8 & 0 \\
\hline comme & 10 & 1 \\
\hline depuis & 10 & 0 \\
\hline mais & 11 & 8 \\
\hline plus & 14 & 5 \\
\hline pour & 19 & 8 \\
\hline
\end{tabular}

* Les verbes ont été lemmatisés, tous les temps ayant été ramenés à l'infinitif.

Même si on multipliait par 3 les données lexicales du discours du Parti québécois pour rétablir une juste proportion, on constaterait aisément que le vocabulaire économique est la marque de commerce du Parti libéral. Ainsi, les mots "économie, emplois, dollars, investissements, croissance" sont fréquemment utilisés alors qu'ils sont pour la plupart absents du discours du Parti québécois.

On remarque aussi que le message libéral est axé sur le bilan de l'action gouvernementale comme en témoigne l'emploi fréquent des mots : «ans, bilan, gouvernement" et de l'adverbe "depuis". Mais ce discours est aussi orienté vers l'avenir, ce mot étant repris 29 fois dans le slogan du parti, qui était "Assurons notre avenir". Les stratèges libéraux ont aussi misé sur la stabilité économique par l'emploi répété du verbe "assurer" qui est lié à "l'avenir», ces deux termes étant les éléments clés du slogan libéral. 
Tableau V : Les mots les plus caractéristiques du discours libéral

\begin{tabular}{|c|c|c|c|c|c|c|c|}
\hline \multicolumn{2}{|c|}{$\begin{array}{l}\text { LIBELLEE DE LA } \\
\text { FORME } \\
\text { GRAPHIQUE }\end{array}$} & \multicolumn{2}{|c|}{ POURCENTAGE } & \multicolumn{2}{|c|}{ FRÉOUENCE } & \multirow[t]{2}{*}{$\begin{array}{l}\text { V. } \\
\text { TEST }\end{array}$} & \multirow[t]{2}{*}{$\begin{array}{l}\text { PRO- } \\
\text { BABI- } \\
\text { LITE }\end{array}$} \\
\hline & & INTERNE & GLOBAL & INTERNE & GLOBALE & & \\
\hline 1 & LIBÉRAL & 1,38 & 1,04 & 30 & 30 & 3,514 & .000 \\
\hline 2 & NOTRE & 1,28 & 1,01 & 28 & 29 & 2,742 & .003 \\
\hline 3 & AVENIR & 1,33 & 1,08 & 29 & 31 & 2,324 & .010 \\
\hline 4 & ANNEEES &, 69 &, 52 & 15 & 15 & 2,169 & .015 \\
\hline 5 & $\begin{array}{l}\text { INVESTIS- } \\
\text { SEMENTS }\end{array}$ & .55 & .42 & 12 & 12 & 1,814 & .035 \\
\hline 6 & AURAIT &, 55 & .42 & 12 & 12 & 1,814 & .035 \\
\hline 7 & ASSURER & .55 & .42 & 12 & 12 & 1,814 & .035 \\
\hline 8 & DOLLARS &, 50 & ,38 & 11 & 11 & 1,683 & ,046 \\
\hline 9 & JEUNES & .73 &, 59 & 16 & 17 & 1,591 & .056 \\
\hline 10 & QUÉBÉCOISE & .46 & .35 & 10 & 10 & 1,546 & .061 \\
\hline 11 & MARCHE & .46 & .35 & 10 & 10 & 1,546 & .061 \\
\hline 12 & VOTONS & .46 & .35 & 10 & 10 & 1,546 & .061 \\
\hline 13 & BILAN & .41 & .31 & 9 & 9 & 1,400 & 081 \\
\hline 14 & ÉCONOMIE & .83 & .69 & 18 & 20 & 1,274 & 101 \\
\hline 15 & BEAUCOUP & .37 & .28 & 8 & 8 & 1,243 & , 107 \\
\hline 16 & ASSURONS & ,37 & ,28 & 8 & 8 & 1,243 & 107 \\
\hline 17 & ROUE & .37 & .28 & 8 & 8 & 1,243 & 107 \\
\hline 18 & IMPOTSS & .37 & .28 & 8 & 8 & 1,243 & 107 \\
\hline 19 & DEPUIS &, 37 &, 28 & 8 & 8 & 1,243 & 107 \\
\hline 20 & EQUIPE & .60 & .49 & 13 & 14 & 1.226 & 110 \\
\hline
\end{tabular}

Les mots suivants ont été retirés de cette liste: à, une, sur, et, des, ces, avec, dans, qui, aussi.

En plus des divergences déjà observées au moyen de l'analyse comparative du vocabulaire, il faut remarquer ici I'utilisation différente des substantifs et des adjectifs «libéraux, libéral, québécois, québécoise". Pour désigner le Parti libéral, le Parti québécois utilise l'adjectif substantivé "les libéraux», désignation contenant une nuance péjorative. Le PLQ pour sa part associe l'adjectif ulibéral» au gouvernement, au parti, à l'économie ou encore à la jeunesse. 
Tableau VI : Les mots les plus caractéristiques du discours péquiste

\begin{tabular}{|c|c|c|c|c|c|c|c|}
\hline \multirow{2}{*}{\multicolumn{2}{|c|}{$\begin{array}{l}\text { LIBELLÉ DE LA } \\
\text { FORME } \\
\text { GRAPHIQUE }\end{array}$}} & \multicolumn{2}{|c|}{ POURCENTAGE } & \multicolumn{2}{|c|}{ FRÉQUENCE } & \multirow{3}{*}{$\begin{array}{c}\text { V. } \\
\text { TEST }\end{array}$} & \multirow{3}{*}{$\begin{array}{l}\text { PRO- } \\
\text { BABI- } \\
\text { LITE } \\
, 000\end{array}$} \\
\hline & & \multirow{2}{*}{$\frac{\text { INTERNE }}{4,54}$} & \multirow{2}{*}{$\frac{\text { GLOBAL }}{1,48}$} & \multirow{2}{*}{$\frac{\text { INTERNE }}{34}$} & \multirow{2}{*}{$\frac{\text { GLOBALE }}{45}$} & & \\
\hline 1 & PARTI & & & & & & \\
\hline 2 & JE & 3,34 & 1,18 & 25 & 36 & 5,544 & .000 \\
\hline 3 & PRENDS & 1,60 &, 39 & 12 & 12 & 5,342 & , 000 \\
\hline 4 & PRENDRE & 1,34 &, 33 & 10 & 10 & 4,805 & , 000 \\
\hline 5 & LAISSER & 1,20 & ,30 & 9 & 9 & 4,514 & .000 \\
\hline 6 & VOTE & 1,07 &, 26 & 8 & 8 & 4,205 & .000 \\
\hline 7 & LIBÉRAUX & 1,07 & ,26 & 8 & 8 & 4,205 & .000 \\
\hline 8 & QUÉBÉCOIS & 1,20 &, 43 & 9 & 13 & 3,139 & ,001 \\
\hline 9 & PARLE & .93 &, 30 & 7 & 9 & 3,039 & .001 \\
\hline 10 & ME & .93 & ,30 & 7 & 9 & 3,039 & .001 \\
\hline 11 & ILS & .80 &, 23 & 6 & 7 & 3,034 & ,001 \\
\hline 12 & QUÉBEC & 4,41 & 2,79 & 33 & 85 & 2,840 & .002 \\
\hline 13 & FAIRE & 2,14 & 1,08 & 16 & 33 & 2,823 & .002 \\
\hline 14 & EMPLOI & .80 & .26 & 6 & 8 & 2,667 & .004 \\
\hline 15 & A & 3,74 & 2,63 & 28 & 80 & 2,003 & .023 \\
\hline 16 & ON & 3,60 & 2,89 & 27 & 88 & 1,211 & .113 \\
\hline 17 & PEUT & 1,07 &, 62 & 8 & 19 & 1,007 & 157 \\
\hline 18 & BIEN & .43 & ,21 & 3 & 6 & ,998 & .159 \\
\hline 19 & SOMMES & .43 & .28 & 3 & 8 & .510 &, 305 \\
\hline 20 & DOIT & .43 & .28 & 3 & 8 & .510 & ,305 \\
\hline
\end{tabular}

Les mots suivants ont été retirés de cette liste: ou, ce, du, se, un, ça, les, la, ne.

L'analyse des segments le plus souvent répétés nous indique que l'adjectif "québécois", dans le discours libéral, est accolé à des éléments économiques tels que "produits" ou «investisseurs", alors que cet adjectif sert seulement à identifier le parti dans le discours de son adversaire. L'emploi du mot Québec est également particulier dans le discours du Parti québécois qui non seulement le répète plus souvent, mais s'en sert pour se présenter comme le parti du Québec, le parti du Québec souverain. Pour sa part, le Parti libéral associe le mot Québec au gouvernement.

La personnalisation du message

Qui est l'émetteur du message ? Sur qui les partis misent-ils pour convaincre? Afin d'évaluer le degré de personnalisation du message, nous utilisons comme indicateurs l'usage des pronoms et la fréquence d'intervention des locuteurs. 
Comme l'indique le tableau IV, les messages du Parti québécois étaient fortement personnalisés, la première personne du singulier ayant été la forme pronominale la plus employée ( 25 fois contre seulement 11 au PLQ). Dans les messages du Parti libéral, c'est le pronom "nous" qui prédomine (46 fois contre 15 pour le Parti québécois). Cette référence au "nous" visait à renforcer l'image d'équipe que cherchait à projeter le Parti libéral qui préférait mettre en évidence certains ministres ou certains candidats considérés comme plus électoralement rentables.

Le tableau VII montre la façon dont chaque parti a réparti la prise de parole.

Tableau VII : Les émetteurs

\begin{tabular}{||l|c|r|c|c||}
\hline \multirow{2}{*}{} & \multicolumn{2}{|c|}{ Parti libéral } & \multicolumn{2}{c||}{ Parti québécois } \\
\cline { 2 - 5 } & $\mathrm{N}^{*}$ & \multicolumn{1}{c|}{$\%$} & $\mathrm{~N}^{*}$ & $\%$ \\
\hline Chef & 44 & 25,7 & 34 & 44,2 \\
Téte d'affiche & 84 & 49,1 & 16 & 20,8 \\
Voix & 10 & 5,8 & 12 & 15,6 \\
Chanson & 21 & 12,3 & 3 & 3,9 \\
Slogan & 12 & 7,0 & 12 & 15,6 \\
Total & 171 & & 77 & \\
\hline
\end{tabular}

*Nombre de phrases prononcées.

Ce relevé indique que la présence du chef libéral se fait plus discrète que celle du chef péquiste. Cette particularité est encore plus évidente dans la publicité sur le réseau anglais où le chef libéral n'apparaît jamais, caché par son parti. Les stratèges libéraux ont préféré donner la parole aux ministres ou aux candidats vedettes, qui accaparent presque $50 \%$ du discours publicitaire. Les ministres Daniel Johnson et Gil Rémillard ont été les plus visibles, suivis par Christiane Pelchat, Michel Pagé, GérardD. Lévesque, Violette Trépanier, Liza Frulla-Hébert, Robert Dutil et Louise Robic. La stratégie inverse a été adoptée par le Parti québécois qui a donné plus de visibilité à son chef qu'à son équipe et réservé seulement $18 \%$ du discours aux autres locuteurs: Pauline Marois, David Cliche, Rémi Trudel, Carmen Juneau, Jacques Brassard. 


\section{Types de messages et fonction partisane}

Notre hypothèse était que la position d'un parti dans le système partisan détermine le contenu de son discours. La fonction gouvernementale oblige donc le parti au pouvoir à centrer son message sur le bilan de son action; la fonction d'opposition incite le parti qui l'assume à critiquer le gouvernement sortant. Pour évaluer cette dimension de la stratégie de communication, nous avons relevé le nombre de phrases consacrées à chacune des composantes des messages.

Tableau VIII : Répartition des composantes des messages

\begin{tabular}{||l|r|r|r|r||}
\hline \multirow{2}{*}{} & \multicolumn{2}{|c|}{ Parti libéral } & \multicolumn{2}{c||}{ Parti québécois } \\
\cline { 2 - 5 } & $\mathrm{N}$ & $\%$ & $\mathrm{~N}$ & $\%$ \\
\hline Jugement de fait & 30 & 17,5 & 11 & 14,3 \\
Objectifs & 39 & 22,8 & 19 & 24,7 \\
Performance passée & 57 & 33,3 & 1 & 1,3 \\
Qualités chef-parti & 8 & 4,7 & 7 & 9,0 \\
Critiques adversaire & 5 & 3,0 & 13 & 16,9 \\
Appel aux électeurs & 8 & 4,7 & 8 & 10,4 \\
Slogans & 24 & 14,0 & 18 & 23,4 \\
& & & & \\
Total & 171 & & 77 & \\
\hline
\end{tabular}

* Les chiffres sous cette rubrique ne correspondent pas à ceux du tableau précédent parce qu'ils incluent les slogans prononcés par le chef ou par une vedette du parti.

À part l'insistance égale que mettent les deux partis sur les faits et sur leurs objectifs, la structure de leur discours se différencie nettement.

Comme il se doit, le parti au pouvoir a surtout fait prévaloir ses réalisations et donné priorité au bilan de son action gouvernementale. À cet égard, le discours publicitaire libéral insiste sur la réduction du déficit et des impôts, la création de 80000 emplois par année (attribuable à une politique favorable aux investisseurs), la diminution du taux de chômage chez les jeunes, la croissance des exportations agricoles aux États-Unis. Toujours au chapitre de ses réalisations, le parti gouvernemental a tenté de valoriser également sa politique environnementale en 
prétendant avoir doublé le budget consacré à l'environnement. Différentes personnalités du parti ont rappelé les avantages de certains programmes comme l'aide à la famille, l'aide aux personnes handicapées, la lutte contre la drogue. On a aussi vanté I'entente du Lac Meech, qui aurait permis au Québec de récupérer son droit de veto.

Sur le plan des engagements, les mots clés sont "stabilité", "croissance» et "prospérité économique". Le Parti libéral promet d' «assurer l'avenir" et de faire tourner la roue de l'économie dont dépendent la qualité de vie et la création d'emplois. II se propose d'atteindre les objectifs suivants : réduire les impôts, stimuler les investissements, protégerl'environnement, encouragerla recherche et le développement technologique, investir dans la formation de la main-d'œuvre, assurer l'intégration des immigrants à la collectivité francophone, protéger les jeunes contre les trafiquants de drogue.

Pour atteindre ces objectifs, le Parti libéral présente «une équipe compétente, qui a fait ses preuves(...) une équipe solide, bien préparée, expérimentée et qui agit avec réalisme et d'une façon concrète». On laisse évidemment entendre que l'adversaire péquiste ne peut posséder ces qualités puisqu'il a laissé le Québec dans une situation dramatique à la fois sur le plan économique et constitutionnel, étant responsable de la perte du droit de veto en 1981-1982. On laisse aussi entendre qu'il menace la stabilité politique et la prospérité économique des Québécois avec son projet d'accéder à l'indépendance par une série de référendums.

Le discours publicitaire du Parti québécois est antinomique, ce parti ayant adopté une stratégie d'attaque basée sur la dénonciation des méfaits de la gestion libérale. Ses messages critiquent le manque de vision, l'indécision et le laisser-faire du gouvernement dans plusieurs secteurs considérés comme des enjeux majeurs de la campagne. En agriculture, les libéraux, disent les péquistes, ont fait perdre 29000 emplois. Ils ne se sont pas occupés de la gestion des déchets toxiques et de l'assainissement des eaux. Ils ont fait des concessions linguistiques et amoindri la portée de la loi 101. Ils n'ont pas aidé généreusement les jeunes familles. Ils ont laissé les services de santé se dégrader. Ils $n^{\prime}$ ont pas protégé les intérêts du Québec dans le dossier constitutionnel. Devant un tel bilan, le choix est clair : "On peut les laisser faire ou prendre parti. Je prends le parti du Québec. Je vote Parti québécois".

Au premier rang de ses objectifs, le Parti québécois place la souveraineté du Québec. II propose d'élire un gouvernement qui 
va préparer la souveraineté et qui va changer des choses. Jacques Parizeau déclare qu'il est entré en politique uniquement pour réaliser la souveraineté du Québec et qu'il s'est fixé comme but de laisser à ses enfants et à ses petits-enfants "un Québec vert et prospère, socialement plus solidaire à l'intérieur de ce cadre de la souveraineté». Rétablir la paix linguistique, intégrer les immigrants, améliorer la qualité de l'enseignement, créer une économie de plein-emploi, tels seront les mots d'ordre d'un gouvernement péquiste. Et pour ce faire, le Parti québécois compte sur une équipe décidée qui offre "des solutions concrètes et efficaces", une équipe qui prend le parti du Québec.

La conclusion du message péquiste est construite sur l'opposition entre deux attitudes : laisser faire ou prendre parti. Une fois cette antinomie posée, on lance un jeu de mots, qui associe l'idée de prendre le parti du Québec au fait de voter pour le Parti québécois.

\section{Conclusion}

L'analyse du vocabulaire a ses limites. Elle ne permet pas de saisir toutes les dimensions de la publicité électorale qui, en plus des mots, fait appel au son et à l'image. L'expression «une image vaut mille mots" le traduit bien d'ailleurs. On a même réalisé, aux États-Unis, des publicité électorales sans une seule parole ${ }^{16}$. II peut arriver aussi que le silence ou l'absence d'un mot soient très significatifs et aient une portée émotive très forte. Dans le message du Parti québécois, qui dénonce la politique linguistique du Parti libéral, le geste supplée aux mots : les Québécois ont reçu la loi 178 comme une giffle. Ainsi, le message avait un contenu agressif qui ne paraît pas à l'analyse du vocabulaire.

Malgré ses limites intrinsèques, l'analyse lexicale, en utilisant le discours comme un révélateur des comportements politiques, permet de documenter de façon rigoureuse et systématique certains phénomènes propres à la communication politique. C'est dans cette perspective que nous avons testé des hypothèses relatives à la personnalisation du pouvoir, au mimétisme politique et aux contraintes stratégiques qu'imposent les positions des partis dans le système partisan.

16. Le sénateur Humphrey s'est permis de diffuser en 1976 un message sans paroles où il apparaissait à l'écran dans un décor bucolique en compagnie de son épouse et sur une trame sonore de musique classique. 
La politique québécoise n'échappe pas au phénomène de la personnalisation du pouvoir. On a pu le constater quand PierreMarc Johnson, lors des élections de 1985, avait presque fait disparaître la référence au Parti québécois dans la publicité électorale, dont le slogan était : "Avec Johnson(...)». En 1989, on a pu observer, par l'analyse des locuteurs et des pronoms, que ce phénomène était récurrent et était plus accentué au Parti québécois qu'au Parti libéral, le premier présentant son chef seul et en gros plan, et le second montrant Robert Bourassa le plus souvent entouré de son équipe. Toutefois, en raison des contraintes imposées par le système parlementaire, cette tendance est contrebalancée par les références àl'identification partisane. Ainsi, le discours publicitaire du Parti libéral fait 10 fois référence au parti et 11 fois au gouvernement libéral; dans le discours du Parti québécois, le nom du parti est mentionné à 9 reprises.

La personnalité des chefs ne semble pas être un atout suffisant pour persuader l'électeur de donner son soutien à un parti; elle doit être supportée par l'idéologie du parti qui est un facteur de choix plus fiable. À cet égard, nous avons constaté que la publicité électorale reflète les divergences idéologiques qui opposent les forces politiques québécoises. La comparaison des vocabulaires nous a permis d'observer que les messages publicitaires traduisent les clivages idéologiques entre les partis. Ainsi, le vocabulaire économique caractérise le discours publicitaire du Parti libéral tandis que le vocabulaire du Parti québécois est plus centré sur l'identité et l'appartenance au Québec. Le Parti québécois, cette fois-ci, a fait la promotion de son objectif fondamental : la souveraineté du Québec. Le Parti libéral, lui, a consacré un message à démontrer les dangers que le projet du Parti québécois faisait courir à la sécurité économique des Québécois. Si le positionnement des deux partis se différencie nettement en ce qui concerne l'enjeu constitutionnel et linguistique, sur les autres enjeux, il est moins tranché. Libéraux et péquistes partagent certains objectifs communs : protection de l'environnement, intégration des immigrants, amélioration de l'éducation et aide à la famille.

Les discours publicitaires diffèrent non seulement par leur contenu, mais aussi par leur structure. Alors que le Parti libéral a opté pour une stratégie basée sur la valorisation de ses réalisations, le Parti québécois a centré sa tactique sur la critique de son adversaire. Cette logique polémique semble correspondre aux contraintes qu'impose la position occupée dans le système partisan : le parti au pouvoir se doit de dresser le bilan de son 
action gouvernementale, et le parti d'opposition d'exercer sa fonction de critique. 